\title{
Coexistence of metastatic thyroid cancer and tuberculosis in a single neck lymph node: a rare association
}

\author{
Kyueng-Whan $\mathrm{Min}^{1}$, Dong-Hoon $\mathrm{Kim}^{2}$, Seoung Wan Chae ${ }^{2}$, Jin Hee Sohn², and Kyoung Min Moon ${ }^{3}$
}

\begin{abstract}
${ }^{1}$ Department of Pathology, Hanyang University Guri Hospital, Hanyang University College of Medicine, Guri, Korea; ${ }^{2}$ Department of Pathology, Kangbuk Samsung Hospital, Sungkyunkwan University School of Medicine, Seoul; ${ }^{3}$ Department of Internal Medicine, Asan Medical Center, University of Ulsan College of Medicine, Seoul, Korea
\end{abstract}

A 31-year-old man was admitted to the outpatient department with an enlarged neck mass that had been growing gradually over the previous month. His medical history was unremarkable, but his mother received anti-tuberculosis medication due to cervical tuberculous lymphadenopathy 10 years ago. Ultrasonography revealed an irregular hypoechoic nodule with calcifications, measuring $1 \mathrm{~cm}$ in diameter in the thyroid and multiple enlarged lymph nodes along the jugular chain of the neck. A malignant thyroid nodule with either Kikuchi disease, tuberculosis or metastasis from other lymph nodes was suspected (Fig. 1).

Fine needle aspiration cytology was performed. The thyroid nodule was suspicious for papillary thyroid carcinoma (PTC). Needle biopsy of lymph node revealed chronic granulomatous inflammation consistent with tuberculosis. Upon Ziehl Neelsen carbo-fuchsin staining, a few acid-fast bacilli were identified. Nested polymerase chain reaction (PCR) amplifications were performed using Mycobacterium tuberculosis IS6110 primers (MTBPCR kit, Biosewoom, Seoul, Korea). PCR detected Mycobacterium tuberculosis (Fig. 1B, inset). Chest X-rays were unremarkable. Tuberculosis culture was not performed. An anti-tuberculosis HERZ (isoniazid [INH], rifampin [RFP], ethambutol $[\mathrm{EMB}]$, and pyrazinamide $[\mathrm{PZA}])$ regimen was started and continued for 6 months.
Received: March 25, 2016

Revised : May 2, 2016

Accepted: February 13, 2017

\section{Correspondence to}

Dong-Hoon Kim, M.D.

Tel: +82-2-2001-2392

Fax: +82-2-2001-2398

E-mail: idavid.kim@samsung.com
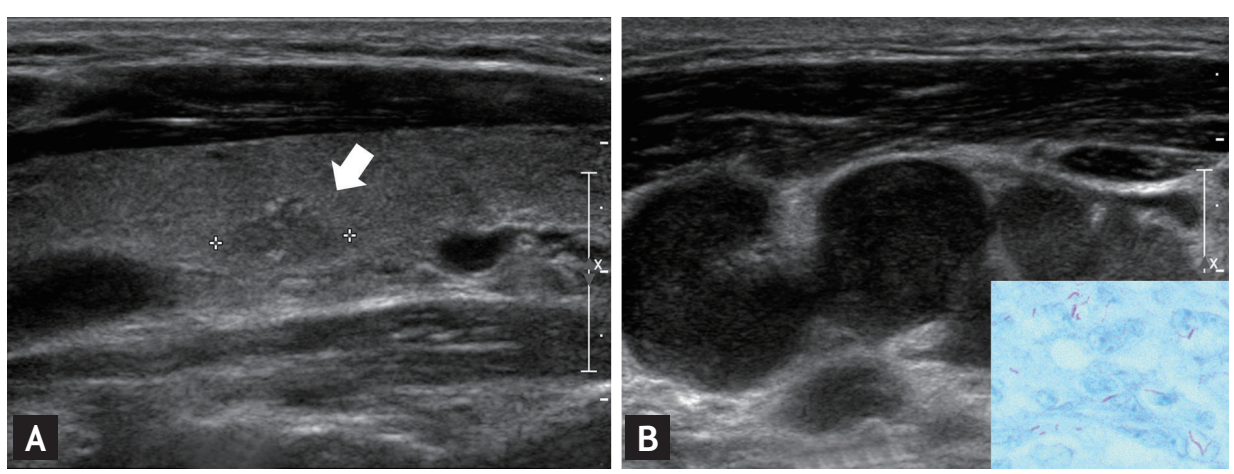

Figure 1. In sonographic imaging, (A) there is an irregular shaped hypoechoic nodule with internal calcifications at the mid pole (white arrow) and (B) multiple enlarged lymph nodes, ranging from 0.5 to $1 \mathrm{~cm}$ along the jugular chain of the neck. In microscopic view of needle biopsy, a few bacilli are noted (inset: Ziehl Neelsen, $\times 1,000$ ). 


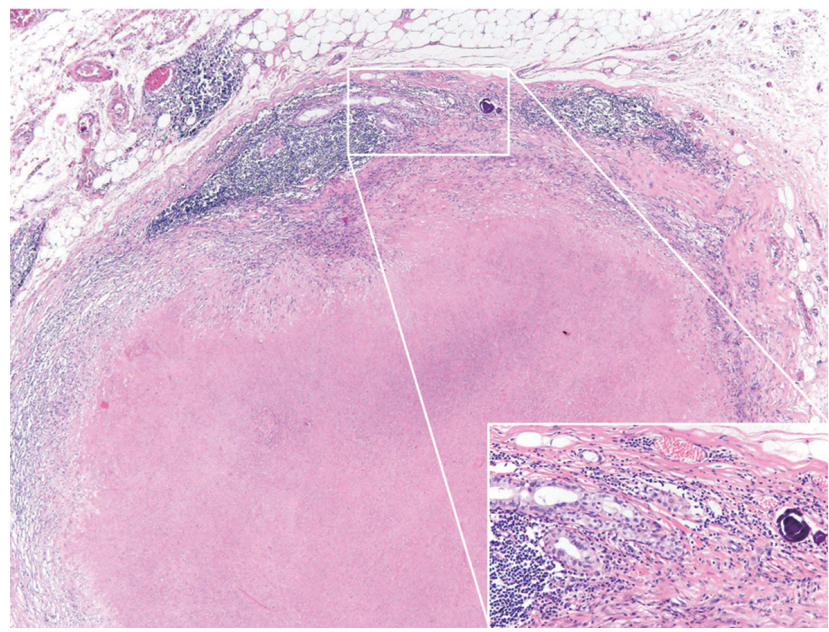

Figure 2. Microscopic view of the lymph node reveals a small number of metastatic papillary thyroid carcinomas present at the periphery of chronic granulomatous inflammation with caseous necrosis (H\&E, ×12.5). Lymph node structures around metastatic papillary thyroid carcinoma with psammoma bodies are preserved (inset: H\&E, ×200).

Computerized tomography was performed after starting medication, and it revealed few lymph nodes that remained swollen. Subsequently, a right hemithyroid- ectomy with neck dissection was performed and was confirmed as PTC. Intriguingly, metastatic PTC was associated with chronic granulomatous inflammation in a single lymph node. Upon whole view examination of the lymph node, lymph node structures were seen at the periphery of the metastatic PTC (Fig. 2). At 2-month postoperative follow-up visit, no complications were observed, and the patient remained well.

Generally, cervical lymphadenopathy originates from metastasis or infection, and metastasis and infection have been identified as main causative factors for lymphadenopathy. The simultaneous occurrence of tuberculosis and metastatic carcinoma is unusual without pulmonary or thyroid tuberculosis. Synchronous dual occurrence of tuberculosis and metastatic carcinoma is often confusing for clinicians, because its presentation is rare. When lymph nodes are continuously enlarged in spite of receiving anti-tuberculosis therapy, the possibility of coexistent metastasis or other malignancies shall not be overlooked.

\section{Conflict of interest}

No potential conflict of interest relevant to this article was reported. 